Instituto Internacional de Investigación y Desarrollo Tecnológico Educativo INDTEC, C.A.

DOI: https://doi.org/10.29394/Scientific.issn.2542-2987.2018.3.7.1.17-36

OAI-PMH: http://www.indteca.com/ojs/index.php/Revista Scientific/oai

\title{
Ambiente Externo y Desempeño Financiero en el Sector Privado de la Salud
}

Autor: Adolfo Javier Cegarra Acosta Universidad Nacional Experimental de los Llanos Occidentales Ezequiel Zamora, UNELLEZ quantum.analitica2@gmail.com

Barinas, Venezuela

\section{Resumen}

El propósito de este estudio es analizar la relación entre el ambiente externo y el desempeño financiero, en el contexto del sector privado de la salud en la ciudad de Barinas (Venezuela). Mediante la revisión de la literatura y un focus group se identificaron 15 factores ambientales que influyen sobre este sector empresarial. Posteriormente, se encuestó a los presidentes de las 25 empresas existentes en Barinas, para medir sus percepciones sobre estos factores ambientales, en cuanto a sus dimensiones de Dinamismo y Munificencia. Los resultados obtenidos en la encuesta y la rentabilidad de cada empresa en su último ejercicio económico, se analizaron con procedimientos de Escalamiento Óptimo. Los hallazgos demostraron la existencia de una fuerte relación entre 12 factores ambientales y la rentabilidad, en el cual el ambiente externo se caracteriza por tener bajo dinamismo (baja variabilidad / alta predictibilidad), en un Entorno de Trabajo munificente y un Entorno General desfavorable.

Palabras clave: planificación estratégica; ganancia; centro médico; empresa privada. 


\title{
External Environment and Financial Performance in the Private Health Sector
}

\begin{abstract}
The purpose of this study is to analyze the relationship between the external environment and financial performance, in the context of the private healthcare sector in Barinas (Venezuela). A review of the literature and a focus group identified 15 environmental factors that influence this business sector. Subsequently, the presidents of the 25 companies in Barinas were surveyed to measure their perceptions about these environmental factors, in terms of their Dynamism and Munificence dimensions. The results obtained in the survey and the profitability of each company in its last financial year were analyzed with Optimal Scaling procedures. The findings demonstrated the existence of a strong relationship between 12 environmental factors and profitability, in which the external environment is characterized by a great complexity, with low dynamism (low variability / high predictability), in a munificent task environment and an unfavorable general environment.
\end{abstract}

Keywords: strategic planning; profits; medical centres; private enterprises. 


\section{Introducción}

En Venezuela, los factores políticos, económicos y sociales del ambiente externo parece que ejercen una influencia significativa sobre el desempeño financiero de las empresas del sector privado de la salud, lo cual se evidenció en un resultado financiero negativo en algunas importantes empresas en el estado Barinas. En líneas generales, parece que existe la percepción de que factores ambientales como la confrontación política entre el gobierno y la oposición, la escasez de medicamentos, equipos y repuestos, la elevada inflación, el aumento de los costos del personal por la vía de decretos presidenciales, entre otros, tienen un efecto muy negativo sobre los indicadores financieros de estas empresas. Esta percepción tiene su fundamento, pues la interferencia política y las variaciones en la situación económica se han relacionado al mal desempeño organizacional, incluso con una estrategia perfectamente formulada (Njoroge, Ongeti, Kinuu y Kasomi, 2016a). Esto conlleva a estudiar la relación ambiente externo-desempeño financiero, sobre la cual ya existe una abundante perspectiva teórica desarrollada desde principio de la década de 1980, con abundantes demostraciones empíricas en los países desarrollados. A pesar de ello, existe una carencia de literatura en los países en desarrollo y, más aún, que aporte evidencia sobre cómo influyen los factores ambientales que se manifiestan concretamente en Venezuela en la viabilidad financiera de las empresas.

La influencia del ambiente externo en el desempeño de las empresas ha sido abordada teoréticamente desde dos perspectivas: la primera de ellas considera que el ambiente externo es una variable que modera la relación entre la estrategia de la empresa y su desempeño organizacional. Este enfoque sugiere que, con el propósito de defender su posición de mercado y desempeño del negocio, las compañías deliberadamente seleccionan estrategias en respuesta a sus ambientes externos (Balas, Gokus y Colakoglu, 2014). 
La segunda perspectiva trata al desempeño organizacional como una variable dependiente, y al ambiente externo como uno de sus constructos independientes (Murgor, 2014). Esta investigación se realizará bajo esta perspectiva, puesto que la totalidad de las organizaciones del sector privado de la salud carecen de una estrategia formal, por una parte, y por la otra, es necesario dilucidar el verdadero papel que juegan los factores ambientales en el desempeño de las empresas del sector, y más concretamente en el desempeño financiero.

En este estudio se pretende identificar los factores ambientales objetivos que influyen sobre las organizaciones privadas de la salud en el contexto de la ciudad de Barinas (Venezuela), así como también describir las percepciones que existen acerca de dichos factores entre los presidentes de estas empresas para, finalmente, correlacionar estos resultados con la rentabilidad alcanzada por estas organizaciones en su último ejercicio económico.

\section{Revisión de la Literatura}

\subsection{El Ambiente Externo}

En la literatura existe un amplio consenso sobre la definición de ambiente externo. Los académicos coinciden en definirlo como una cierta combinación de factores, fuera de los límites de la organización que influyen el desempeño de sus actividades (Krivstov, 2014a). Todas las organizaciones son sistemas abiertos (Njoroge et al., 2016b), que están en constante intercambio con sus ambientes. El ambiente externo proporciona a las empresas insumos que transforman en productos a través de procesos internos y luego los productos se devuelven al medio ambiente. Las organizaciones no pueden tener control sobre los factores ambientales. El medio ambiente es fuente de restricciones, contingencias, problemas y oportunidades que afectan los términos en los que las organizaciones hacen 
negocios.

Contrariamente, se encuentra un extenso desacuerdo sobre cómo debe evaluarse y medirse el ambiente externo. Algunos teóricos tratan al ambiente externo como hechos objetivos independientes de las empresas mientras que otros investigadores consideran que el ambiente externo está determinado por las percepciones de sus actores. Sin embargo, Bourgeois, (citado por Njoroge et al., 2016c), concluyó que la cuestión no era si las medidas debían ser objetivas o perceptivas; más bien, sugirió que tanto las medidas objetivas como las medidas perceptuales son reales y relevantes para la estrategia de una organización. Más aun, argumenta que la diferencia en la visión conceptual entre las medidas objetivas y las medidas perceptuales no parece ser distintiva. En este contexto, muchos estudios encontraron una fuerte correlación entre evaluaciones perceptivas y objetivas del medio ambiente. Tomando en cuenta estos puntos de vista, operacionalizó el ambiente externo en dos dimensiones: el Ambiente Objetivo, formado por los factores ambientales externos; y el Ambiente Percibido, constituido por las percepciones internas que se tienen sobre dichos factores ambientales. A su vez, el Ambiente Objetivo está formado por el Entorno General y el Entorno de Trabajo.

Para Krivstov (2014b), el Entorno General lo conforman las condiciones comunes para el funcionamiento de todas las organizaciones $\mathrm{y}$, por regla general, no tiene rasgos peculiares en relación con alguna empresa en particular, sin embargo, el nivel de su influencia en las diferentes empresas puede ser diferente. El Entorno General lo constituyen los factores políticos, legales, económicos, tecnológicos, sociales y ambientales.

Por otra parte, el estudio del Entorno de Trabajo de una empresa tiene como objetivo evaluar aquellos factores ambientales que están en una interacción más directa con cada organización. Éste se basa en el estudio del ambiente de negocios, lo cual incluye proveedores, compradores, 
subcontratistas, clientes, inversores, acreedores, competidores y demás terceros interesados, bien sea a nivel empresarial, a nivel corporativo o a nivel del sector empresarial, según sea el caso (Krivstov, 2014c, pág. 31).

El ambiente externo consta de tres dimensiones 0 patrones subyacentes identificados para evaluar y entender el concepto de ambiente externo de manera sistemática, denominadas Munificencia, Dinamismo y Complejidad (Njoroge, et al., 2016d, pág. 42). La munificencia se refiere a la escasez o abundancia de recursos disponibles en un entorno, y demandados por una o más empresas que operan en un mismo ambiente (Gathungu, Aiko y Machuki, 2014). El dinamismo alude a la naturaleza siempre cambiante del ambiente externo, lo cual puede transformar el propósito de la empresa y el entorno en el que opera (Machuki y Aosa, citado por Njoroge et al., 2016e, pág. 42) y se compone de numerosas variables tales como la velocidad en la que el entorno está cambiando (estable-turbulento) y predictibilidad (predecible-impredecible). Y la complejidad se evalúa a través del número de cuestiones que las organizaciones deben abordar en los diversos aspectos ambientales y si esas cuestiones son similares o diferentes entre sí (Njoroge et al., 2016f, pág. 42).

\subsection{El Desempeño Financiero}

El Desempeño Financiero puede ser interpretado como la medida de qué tan bien la empresa logra sus objetivos financieros. Muchos investigadores insisten en que las medidas financieras son las más razonables para medir el desempeño de una empresa (Affes, 2016a, pág. 1).

Los ingresos y las utilidades son variables importantes para medir el desempeño financiero de una empresa. Al contrastar la utilidad con los ingresos, los activos y el patrimonio de la empresa se obtienen las medidas de rentabilidad. Las medidas tradicionales de rentabilidad para comparar el desempeño financiero entre las empresas han sido el Retorno sobre la 
Inversión (ROI, por sus siglas en inglés), el Retorno sobre las Ventas (ROS) y el Retorno sobre el Patrimonio (ROE) (Affes, 2016b, pág. 2). El Retorno sobre la Inversión, también llamado Rentabilidad Económica, evalúa el beneficio obtenido por la inversión en activos. El Retorno sobre las Ventas o Margen de Utilidad, mide la ganancia derivada de comercialización de los servicios. Y el Retorno sobre el Patrimonio, denominado Rentabilidad del Accionista, computa el rendimiento sobre los aportes de capital de los accionistas.

\section{Métodos}

\subsection{Diseño de la Investigación.}

Este estudio es una generalización empírica que pone a prueba un conjunto de hipótesis para identificar relaciones significativas entre los factores ambientales y la rentabilidad de las empresas, a través de las dimensiones del ambiente externo.

Se utilizó un diseño transeccional pues la información que se recogió sobre los temas sucede en un solo punto en el tiempo. Para los propósitos de este estudio, todas las 25 empresas privadas de salud que existen en la ciudad de Barinas se seleccionaron, por lo que se realizó un censo.

\subsection{Medición de los constructos claves}

3.2.1. El Ambiente Externo. El ambiente externo se operacionalizó a lo largo de dos categorizaciones principales. La primera es la composición del ambiente externo, que se refiere a los factores ambientales objetivos que conforman el entorno focal de la organización. $Y$, en segundo lugar, las dimensiones ambientales, que se refieren a la percepción de los atributos del ambiente del sector. Para evaluar la naturaleza del ambiente externo, se utilizaron ambas categorizaciones, a la vez que se obtuvieron tanto medidas objetivas como perceptuales. Se identificaron los 15 factores ambientales objetivos más relevantes para el sector, sobre aspectos políticos, económicos, 
proveedores y clientes, y se utilizaron dos dimensiones (Munificencia y Dinamismo) para describir las percepciones sobre cada uno de los factores. La dimensión del Dinamismo se midió por la variabilidad y predictibilidad de cada factor ambiental. Por su parte, la Munificencia se midió por la favorabilidad de los factores ambientales sobre las organizaciones, es decir, cuan favorable o desfavorable es cada factor para cada empresa en particular. De esta manera, se preguntó a los encuestados su percepción sobre la variabilidad, predictibilidad y favorabilidad de cada uno de los quince factores ambientales objetivos identificados antes.

3.2.2. Desempeño Financiero. Para medir este constructo, se adoptó el enfoque basado en las medidas de Rentabilidad, de las cuales se prefirieron el ROI para establecer el efecto del ambiente externo sobre la utilización de los activos; y el ROE, con el fin de precisar la rentabilidad del aporte de los accionistas. Para calcular estas medidas, en la encuesta se indagaron los montos de las Utilidades Netas, el Capital Contable y el Activo Total en los estados financieros de las compañías al cierre del ejercicio económico 2016. Con estos datos, se calcularon las medidas de rentabilidad, ROI y ROE, como la proporción de la Utilidad Neta respecto al Activo Total y el Capital Contable, respectivamente.

\subsection{Hipótesis de la investigación}

Se plantearon 4 hipótesis. Las tres primeras someten a prueba las asociaciones entre los factores ambientales y las dimensiones del ambiente externo, Dinamismo y Munificencia. La cuarta hipótesis intenta probar la relación entre estas dimensiones y el desempeño financiero. Las hipótesis por examinar son las siguientes:

H1: Los factores ambientales están relacionados con la Variabilidad del Ambiente Externo. Busca identificar los factores ambientales que influyen en la estabilidad o turbulencia del ambiente externo. 
H2: Los factores ambientales están relacionados con la Predictibilidad del Ambiente Externo. Investiga los factores ambientales asociados con lo predecible o impredecible del ambiente externo.

H3: Los factores ambientales están relacionados con la Favorabilidad del Ambiente Externo. Examina los factores ambientales que intervienen de manera favorable o desfavorable en el ambiente externo.

H4: La Variabilidad, la Predictibilidad y la Favorabilidad del Ambiente Externo están relacionados con la Rentabilidad. Escruta la relación entre las dimensiones del ambiente externo y el desempeño financiero.

\subsection{Recolección de datos.}

Los datos se recolectaron a partir de fuentes primarias y secundarias. Los factores ambientales se identificaron a partir de la revisión del perfil que emitió The Economist Intelligence Unit (EIU) sobre Venezuela en febrero 2017, el cual fue codificado y vinculado con el software de análisis de datos cualitativos Atlas.ti v.7.5.4. De esta manera se acopió un inventario de 75 factores objetivos del Entorno General (políticos y económicos), los cuales se sometieron a la consideración de un focus group formado por 6 expertos del sector privado de la salud, quienes los jerarquizaron de acuerdo con el grado de influencia que perciben sobre el sector, y se seleccionaron los 11 factores que acumularon el $80 \%$ de los votos. Adicionalmente, los miembros del focus group incorporaron 4 factores que pertenecen al Entorno de Trabajo del sector. Como resultado, los 15 factores identificados fueron: transición política (se refiere a la posibilidad de que ocurra una transición política en el gobierno nacional); concentración de poderes (incluye la acumulación de poderes especiales en el poder ejecutivo nacional); tasa de inflación; deterioro de la infraestructura (trata de la desmejora de las instalaciones del sector público de la salud) hospitalaria pública; régimen impositivo; mercado negro de divisas; inamovilidad laboral; escasez (denota la carencia de insumos, equipos y 
repuestos; regulación de precios; control cambiario; sobrevaloración de la moneda; condiciones de compras (describe la negociación de condiciones de compra con los proveedores); poder adquisitivo (alude al poder adquisitivo de aquellos clientes que no están amparados por pólizas de seguros); cobertura de las pólizas (se refiere a los montos cubiertos por las pólizas de seguros y fondos de salud); y deuda de los seguros (se trata de las cuentas por cobrar a las compañías aseguradoras y demás clientes que pagan a crédito los servicios prestados por las empresas del sector).

Se preguntó a los encuestados el grado percibido de variabilidad, predictibilidad y favorabilidad de cada factor ambiental, en una escala de Likert de 5 categorías. El grado de variabilidad y predictibilidad midieron el dinamismo, y el grado de favorabilidad evaluó la munificencia. La confiabilidad fue 0,818 , obtenida con el coeficiente alfa de Cronbach.

\subsection{Técnicas de Análisis.}

Se emplearon técnicas estadísticas de Escalamiento Óptimo para analizar los datos y probar las hipótesis de investigación.

Las 3 primeras hipótesis fueron evaluadas mediante el Análisis de Componentes Principales Categóricos (CATPCA) con rotación varimax. De esta manera, la Variabilidad de los 15 factores ambientales se redujo a 2 componentes ("Variab1" y "Variab2"), cuyas cargas en componentes rotados (que equivalen a las correlaciones de Pearson) revelan la contribución de los factores ambientales en cada componente. Por otro lado, la cuantificación de las 5 categorías (de la escala de Likert) de cada factor ambiental dilucidó el grado de percepción predominante en cada uno de ellos. De la misma manera se procedió con los otros 2 indicadores de las dimensiones del ambiente externo (Predictibilidad y Favorabilidad), y se obtuvieron 2 componentes para cada una de ellas, denominados "Predict1", "Predict2", "Favorab1" y "Favorab2" respectivamente. 
La ROI y la ROE se redujeron mediante un "Análisis Factorial" a un solo componente denominado "Rentab".

Para demostrar las asociaciones significativas entre las dimensiones del ambiente externo y el desempeño financiero se empleó el Análisis de Correlación Canónica No Lineal (OVERALS). Con los 7 componentes obtenidos previamente en los procedimientos CATPCA y factorial, se construyeron 4 conjuntos de datos denominados "Variabilidad", "Predictibilidad", "Munificencia" y "Rentabilidad", y se analizaron con OVERALS, con el cual se obtuvieron las saturaciones, que permitieron determinar cuáles componentes están asociados con la rentabilidad. Todo esto permitió mapear la relación entre los factores ambientales y el desempeño financiero, así como el grado de percepción de cada factor que influye en esta relación.

\section{Resultados}

H1: Los factores ambientales están relacionados con la Variabilidad del Ambiente Externo.

El procedimiento CATPCA de las percepciones de la variabilidad de los factores ambientales arrojó una solución de 2 componentes, denominados "Variab1" y "Variab2", que en conjunto explicaron el $75,9 \%$ de la varianza de los datos. Las cargas de componente rotado revelaron que "Variab1" está asociado a 10 factores ambientales, mientras que "Variab2" correlaciona con los 5 otros factores ambientales.

CATPCA también asignó una cuantificación óptima a las categorías de percepción de variabilidad de cada factor ambiental, cuyo mayor valor absoluto representa la categoría que se diferencia más claramente y, por ende, la que tiene una mayor contribución en la solución obtenida. En la Tabla 1 se identifican las cargas de componentes rotados de los factores ambientales en cada componente, y la cuantificación de la categoría predominante en la 
solución obtenida:

Tabla 1. Variabilidad de los factores ambientales.

\begin{tabular}{|c|c|c|c|c|}
\hline \multirow[t]{2}{*}{ Factor Ambiental } & \multicolumn{2}{|c|}{$\begin{array}{l}\text { Cargas de } \\
\text { componente } \\
\text { rotado }\end{array}$} & \multicolumn{2}{|c|}{$\begin{array}{c}\text { Cuantificación de la Categoría } \\
\text { predominante en cada factor } \\
\text { ambiental }\end{array}$} \\
\hline & $\begin{array}{l}\text { Variab } \\
1\end{array}$ & $\begin{array}{c}\text { Varia } \\
\text { b2 }\end{array}$ & $\begin{array}{c}\text { Valor y Etiqueta de } \\
\text { Categoría }\end{array}$ & $\begin{array}{c}\text { Cuantificació } \\
n\end{array}$ \\
\hline Transición Política & &,- 922 & $5=$ Muy cambiante & 3,667 \\
\hline $\begin{array}{l}\text { Concentración de } \\
\text { poderes }\end{array}$ & ,666 & & $\begin{array}{l}1=\text { Muy poco } \\
\text { cambiante }\end{array}$ & $-1,592$ \\
\hline Tasa de inflación & ,874 & & $\begin{array}{l}1=\text { Muy poco } \\
\text { cambiante }\end{array}$ & $-2,530$ \\
\hline $\begin{array}{l}\text { Deterioro de la } \\
\text { infraestructura }\end{array}$ & & 732 & $\begin{array}{l}1=\text { Muy poco } \\
\text { cambiante }\end{array}$ & $-1,990$ \\
\hline $\begin{array}{l}\text { Régimen } \\
\text { impositivo }\end{array}$ & ,775 & & $\begin{array}{l}1=\text { Muy poco } \\
\text { cambiante }\end{array}$ & $-2,272$ \\
\hline $\begin{array}{l}\text { Mercado negro de } \\
\text { divisas }\end{array}$ & ,882 & & $\begin{array}{l}1=\text { Muy poco } \\
\text { cambiante }\end{array}$ & $-3,716$ \\
\hline $\begin{array}{l}\text { Inamovilidad } \\
\text { laboral }\end{array}$ & & ,939 & $5=$ Muy cambiante & 3,654 \\
\hline Escasez & 910 & & $\begin{array}{l}1=\text { Muy poco } \\
\text { cambiante }\end{array}$ & $-3,577$ \\
\hline $\begin{array}{l}\text { Regulación de } \\
\text { precios }\end{array}$ & 661 & & $\begin{array}{l}1=\text { Muy poco } \\
\text { cambiante }\end{array}$ & $-1,366$ \\
\hline Control cambiario & 542 & & $\begin{array}{l}1=\text { Muy poco } \\
\text { cambiante }\end{array}$ &,- 814 \\
\hline $\begin{array}{l}\text { Sobrevaloración } \\
\text { de la moneda }\end{array}$ & & ,756 & $\begin{array}{l}1=\text { Muy poco } \\
\text { cambiante }\end{array}$ & $-1,799$ \\
\hline $\begin{array}{l}\text { Condiciones de } \\
\text { compra }\end{array}$ & & 817 & 2=Poco cambiante & $-2,223$ \\
\hline Poder adquisitivo & ,782 & & $\begin{array}{l}\text { 1=Muy poco } \\
\text { cambiante }\end{array}$ & $-2,128$ \\
\hline $\begin{array}{l}\text { Coberturas de las } \\
\text { pólizas }\end{array}$ & ,863 & & $\begin{array}{l}1=\text { Muy poco } \\
\text { cambiante }\end{array}$ & $-3,611$ \\
\hline $\begin{array}{l}\text { Deuda de los } \\
\text { seguros }\end{array}$ & ,952 & & $\begin{array}{l}1=\text { Muy poco } \\
\text { cambiante }\end{array}$ & $-3,381$ \\
\hline
\end{tabular}

Fuente: El Autor, (2017). 
H2: Los factores ambientales están relacionados con la Predictibilidad del Ambiente Externo.

También mediante el procedimiento CATPCA, la predictibilidad de los 15 factores ambientales se redujo a 2 componentes, que se denominaron "Predict1" y "Predict2", los cuales explicaron el 76,1\% de la varianza de los datos. De acuerdo con las cargas de componente rotado, "Predict1" está asociado con 10 factores ambientales mientras que "Predict2" se relaciona con los otros 5 factores ambientales. En la Tabla 2 se muestran los resultados de las cargas de componentes rotados y la cuantificación de las categorías de percepción de predictibilidad de cada factor ambiental predominantes en la solución obtenida:

Tabla 2. Predictibilidad de los factores ambientales.

\begin{tabular}{|c|c|c|c|c|}
\hline \multirow[t]{2}{*}{ Factor Ambiental } & \multicolumn{2}{|c|}{$\begin{array}{l}\text { Cargas de } \\
\text { componente } \\
\text { rotado }\end{array}$} & \multicolumn{2}{|c|}{$\begin{array}{l}\text { Cuantificación de la Categoría } \\
\text { predominante en cada factor } \\
\text { ambiental }\end{array}$} \\
\hline & $\begin{array}{l}\text { Predi } \\
\text { ct1 }\end{array}$ & $\begin{array}{l}\text { Predi } \\
\text { ct2 }\end{array}$ & $\begin{array}{c}\text { Valor y Etiqueta de } \\
\text { Categoría }\end{array}$ & $\begin{array}{l}\text { Cuantific } \\
\text { ación }\end{array}$ \\
\hline Transición Política & & ,792 & $5=$ Muy predecible & 1,899 \\
\hline Concentración de poderes & & ,636 & 5=Muy predecible & 1,590 \\
\hline Tasa de inflación & & ,927 & 5=Muy predecible & 1,338 \\
\hline Deterioro infraestructura & ,864 & & $1=$ Impredecible & $-1,650$ \\
\hline Régimen impositivo & ,703 & & $1=$ Impredecible & $-1,600$ \\
\hline Mercado negro de divisas & ,693 & & 4=Predecible & 1,282 \\
\hline Inamovilidad laboral & ,871 & & $1=$ Impredecible & $-1,726$ \\
\hline Escasez & ,826 & & $1=$ Impredecible & $-1,323$ \\
\hline Regulación de precios & ,723 & & $5=$ Muy predecible & 1,315 \\
\hline Control cambiario & ,957 & & $1=$ Impredecible & $-1,456$ \\
\hline $\begin{array}{l}\text { Sobrevaloración de la } \\
\text { moneda }\end{array}$ & ,904 & & $1=$ Impredecible & $-1,501$ \\
\hline Condiciones de compras & &, 914 & 5=Muy predecible & 1,937 \\
\hline Poder adquisitivo & & ,796 & 5=Muy predecible & 1,995 \\
\hline Coberturas de las pólizas & 693 & & $2=$ Poco predecible & $-2,223$ \\
\hline Deuda de los seguros & ,717 & & $1=$ Impredecible & $-2,034$ \\
\hline
\end{tabular}

Fuente: El Autor, (2017). 
H3: Los factores ambientales están relacionados con la Favorabilidad del Ambiente Externo.

El procedimiento CATPCA redujo las percepciones de favorabilidad de los 15 factores ambientales a 2 componentes designados como "Favorab1" y "Favorab2", los cuales explicaron el 70,4\% de la varianza de los datos. Las cargas de componente rotado mostraron la relación de "Favorab1" con 9 factores ambientales. Por su parte, "Favorab2" se relaciona con los otros 6 factores ambientales. En la Tabla 3 que se exhiben a continuación, se presentan los resultados de las cargas de componentes rotados y la cuantificación de las categorías de percepción de favorabilidad preponderantes en cada factor ambiental.

Tabla 3. Favorabilidad de los factores ambientales.

\begin{tabular}{|c|c|c|c|c|}
\hline \multirow[t]{2}{*}{ Factor Ambiental } & \multicolumn{2}{|c|}{$\begin{array}{l}\text { Cargas de } \\
\text { componente } \\
\text { rotado }\end{array}$} & \multicolumn{2}{|c|}{$\begin{array}{l}\text { Cuantificación de la Categoría } \\
\text { preponderante en cada factor } \\
\text { ambiental }\end{array}$} \\
\hline & $\begin{array}{l}\text { Favor } \\
\text { ab1 }\end{array}$ & $\begin{array}{l}\text { Favor } \\
\text { ab2 }\end{array}$ & $\begin{array}{l}\text { Valor y Etiqueta de } \\
\text { Categoría }\end{array}$ & $\begin{array}{l}\text { Cuantifi } \\
\text { cación }\end{array}$ \\
\hline Transición Política & ,-653 & & 2=Desfavorable & $-1,782$ \\
\hline Concentración de poderes & & 902 & $5=$ Muy favorable & 1,774 \\
\hline Tasa de inflación & ,745 & & $3=$ Neutro & 3,559 \\
\hline Deterioro infraestructura & ,726 & & $3=$ Neutro & 2,256 \\
\hline Régimen impositivo & & 768 & $1=$ Muy desfavorable & 1,357 \\
\hline Mercado negro de divisas & ,773 & & 2=Desfavorable & 1,414 \\
\hline Inamovilidad laboral & ,719 & & $3=$ Neutro & 1,464 \\
\hline Escasez & &,- 604 & 1=Muy desfavorable & $-1,265$ \\
\hline Regulación de precios & ,856 & & 4=Favorable & 1,509 \\
\hline Control cambiario & 869 & & $3=$ Neutro & 2,968 \\
\hline Sobrevaloración moneda & ,860 & & $3=$ Neutro & 1,494 \\
\hline Condiciones de compras & & 809 & 4=Favorable & 1,705 \\
\hline Poder adquisitivo & & 875 & $5=$ Muy favorable & 2,579 \\
\hline Coberturas de las pólizas & & 827 & $5=$ Favorable & 2,060 \\
\hline Deuda de los seguros & ,915 & & $3=$ Neutro & 2,901 \\
\hline
\end{tabular}

Fuente: El Autor, (2017). 
H4: La Variabilidad, la Predictibilidad y la Favorabilidad del Ambiente Externo están relacionados con la Rentabilidad.

Con la técnica OVERALS se obtuvo una solución de 2 dimensiones que explicó el $70,7 \%$ de la varianza de los datos.

El análisis de Saturaciones en Componentes que se muestra en la Tabla 4 proporciona una indicación de la contribución que cada componente del ambiente externo aporta a las dimensiones de la solución obtenida. De esta manera, se encontraron altas saturaciones entre los componentes "Variab1", "Predict2", "Favorab2" y "Rentab" en la Dimensión 1; y entre "Variab2", Predict1" y "Favorab1" en la Dimensión 2. Por lo tanto, en la Dimensión 1 se evidenció la relación entre la rentabilidad y los factores ambientales asociados a los componentes "Variab1", "Predict2", "Favorab2".

Tabla 4. OVERALS: Saturaciones en Componentes.

\begin{tabular}{cccc}
\hline \multirow{2}{*}{ Conjunto } & \multirow{2}{*}{ Componente } & \multicolumn{2}{c}{ Saturaciones en componentes } \\
\cline { 3 - 4 } & & Dimensión1 & Dimensión2 \\
\hline \multirow{2}{*}{ Variabilidad } & Variab1 & $-\mathbf{8 6 3}$ &, 120 \\
& Variab2 &, 213 &, 798 \\
\hline \multirow{2}{*}{ Predictibilidad } & Predict1 &, 198 &,- 913 \\
& Predict2 &, $\mathbf{6 3 2}$ &, 686 \\
\hline \multirow{2}{*}{ Munificencia } & Favorab1 &,- 303 &, 831 \\
& Favorab2 & $\mathbf{8 1 4}$ &, 175 \\
\hline Rentabilidad & Rentab & $\mathbf{9 5 1}$ &, 149 \\
\hline Pérdida media & & $\mathbf{1 8 4}$ &, 401 \\
\hline Autovalor & & $\mathbf{8 1 6}$ &, 599 \\
\hline Ajuste & & \multicolumn{3}{c}{1,414} \\
\hline
\end{tabular}

Fuente: El Autor, (2017).

La robustez de la solución obtenida se evaluó a partir de la pérdida media y el autovalor de la dimensión 1, que también se muestran en la Tabla 4. La pérdida media permitió calcular el coeficiente de correlación múltiple de dicha dimensión, con un resultado de 0,903; y la proporción del ajuste real que se explica en esta dimensión se calculó a partir del autovalor, y fue 57,7\%. 
Ambos valores demuestran que existe un elevado grado de relación entre los componentes.

\section{Discusión de Resultados.}

Los hallazgos demostraron la existencia de una fuerte relación entre el ambiente externo y el desempeño financiero y a la vez brinda apoyo a la perspectiva teórica que consideran al desempeño organizacional como una variable dependiente, y al ambiente externo como uno de sus constructos independientes. Ciertamente, se concluyó que el ambiente externo está fuertemente relacionado con el desempeño financiero del sector privado de la salud en el contexto estudiado, pues se encontraron correlaciones muy altas entre la mayoría de los factores ambientales, las dimensiones del ambiente externo y la rentabilidad de las empresas del sector que sugieren la existencia de esta fuerte relación. La hipótesis $\mathrm{H} 4$ se comprobó mediante el procedimiento OVERALS, el cual evidenció una elevada saturación en los componentes "Variab1", "Predict2", "Favorab2" y "Rentab" en la dimensión 1, lo cual sugiere que existe una fuerte relación entre ellos, por lo tanto, los factores ambientales asociados a estos componentes son los que influyen en el desempeño financiero. La identificación de estos factores se logró con la puesta a prueba de las demás hipótesis de la investigación.

El procedimiento CATPCA de las percepciones de Variabilidad puso a prueba la hipótesis $\mathrm{H} 1$, y el análisis de cargas de componente rotado encontró que 10 factores ambientales tienen una contribución manifiesta en el componente "Variab1". Estos factores fueron: concentración de poderes; inflación; régimen impositivo; mercado negro de divisas; escasez; regulación de precios; control cambiario; poder adquisitivo; coberturas de las pólizas; y deuda de los seguros. En todos estos factores la cuantificación predominante fue la correspondiente a la categoría 1 "Muy Poco Cambiante".

De la misma manera, la aplicación de CATPCA a las percepciones de 
Predictibilidad se empleó para poner a prueba la hipótesis H2 y puso de manifiesto que 5 factores ambientales tienen una elevada carga rotada en el componente "Predict2", que los distinguen como los factores que tienen una contribución fundamental en dicho componente, los cuales son: transición política; concentración de poderes; inflación; condiciones de compras; y poder adquisitivo. En estos factores la cuantificación que predominó fue la correspondiente a la categoría 5 "Muy Predecible".

La evidencia también sugiere que los actores del sector perciben que el ambiente externo se caracteriza por tener un bajo dinamismo. En efecto, la cuantificación de los indicadores de variabilidad y predictibilidad ambiental señalaron que las categorías preponderantes en la solución fueron "Muy poco cambiante" y "Muy predecible" respectivamente, lo cual indica que predominó la percepción de que el ambiente externo es estable y predecible, o lo que es lo mismo, poco dinámico. Esta condición resulta conveniente para las empresas del sector pues les otorga una mayor capacidad de reacción ante los cambios que pudieran ocurrir en el medio ambiente.

Finalmente, el empleo de CATPCA en las percepciones de Favorabilidad puso a prueba la hipótesis H3 y evidenció que 6 factores ambientales tienen la mayor contribución en el componente "Favorab2", sin embargo, la cuantificación de las categorías preponderantes en estos factores arrojó resultados divergentes. Por una parte, concentración de poderes, condiciones de compras, poder adquisitivo, y las coberturas de las pólizas se manifestaron como "Favorables/Muy Favorables"; mientras que régimen impositivo y escasez se revelaron "Muy Desfavorables". Estos resultados sugieren que los actores del sector perciben que en el Entorno de Trabajo hay condiciones munificentes para operar exitosamente, mientras que la escasez y los impuestos son factores del Entorno General que constituyen serias amenazas que afectan a las empresas. Las relaciones obtenidas se representaron en la figura 1 que se exhibe a continuación. 
Figura 1: Relaciones entre factores ambientales, dimensiones del ambiente externo y desempeño financiero.

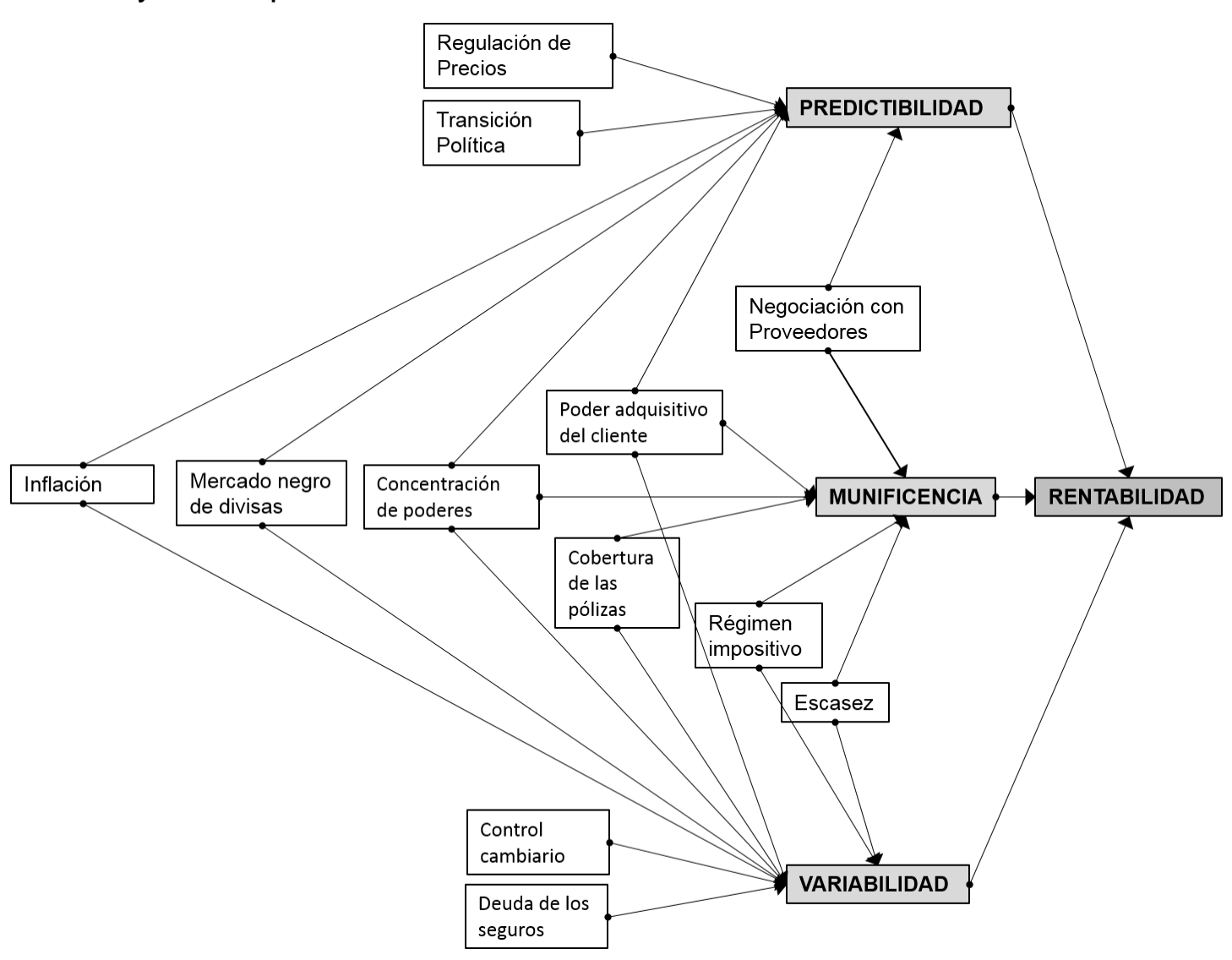
estratégicas más adecuadas. 


\section{Referencias}

Affes, H. (2016a,b). Cognitive role of engineers and its implications on financial performance: An empirical analysis on the french market. Journal of Internet Banking and Commerce, 21(2), 1.

Balas, A. N., Gokus, O., \& Colakoglu, S. N. (2014). Exploring the role of external environment on determining strategic focus, market orientation, and firm performance of service firms. Academy of Marketing Studies Journal, 18(1), 19.

EIU (2017). Country Analysis: Venezuela. The Economist Intelligence Unit. Recuperado de: http://country.eiu.com/Venezuela

Gathungu J., Aiko D. \& Machuki V. (2014). Entrepreneurial Orientation, Networking, External Environment, and Firm Performance: A Critical Literature Review. European Scientific Journal, march vol. 10(7).

Krivstov, A. (2014a,b,c,d). Strategic Analysis of External Environment as a Basis for Risks Assessment. Actual Problems of Economics, \#10(160).

Murgor, P. (2014). External environment, firm capabilities, strategic responses and performance of large scale manufacturing firms in Kenya. University of Nairobi, Unpublished Ph.D. Thesis.

Njoroge J., Ongeti W., Kinuu D., \& Kasomi, F., (2016a,b,c,d,e,f). Does External Environment Influence Organizational Performance? The Case of Kenyan State Corporations. Management and Organizational Studies, 3(3). 


\section{Adolfo Javier Cegarra Acosta}

e-mail: quantum.analitica2@gmail.com

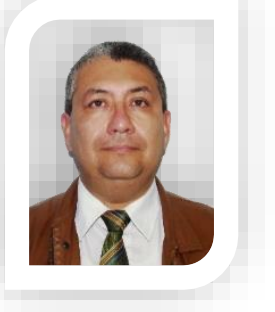

Nacido en Barinas, Venezuela, el 14/06/1963. Ingeniero Agroindustrial, MSc. en Ingeniería Industrial y Especialista en Gerencia Empresarial. Candidato a Doctor en Administración de Empresas en la Universidad Politécnica de Madrid (UPM).

Diez años en la industria nacional de alimentos (HALACA, PROTINAL, REMAVENCA). Diecisiete años dirigiendo empresas del sector privado de la salud. Profesor universitario en el IUP "Santiago Mariño" y la Universidad Santa María. 